\title{
Superimposed Image Description and Retrieval for Fish Species Identification
}

\author{
Uma Murthy ${ }^{1}$, Edward A. Fox ${ }^{1}$, Yinlin Chen ${ }^{1}$, Eric Hallerman ${ }^{2}$, \\ Ricardo da Silva Torres ${ }^{3}$, Evandro J. Ramos ${ }^{3}$, and Tiago R.C. Falcão ${ }^{3}$ \\ 1 Department of Computer Science, Virginia Tech, Blacksburg, VA 24061, USA \\ 2 Department of Fisheries and Wildlife Sciences, Virginia Tech, Blacksburg, \\ VA 24061, USA \\ 3 Institute of Computing, University of Campinas, Campinas, SP, Brazil \\ umurthy,fox,ylchen@vt.edu, ehallerm@vt.edu, rtorres@ic.unicamp.br
}

\begin{abstract}
Fish species identification is critical to the study of fish ecology and management of fisheries. Traditionally, dichotomous keys are used for fish identification. The keys consist of questions about the observed specimen. Answers to these questions lead to more questions till the reader identifies the specimen. However, such keys are incapable of adapting or changing to meet different fish identification approaches, and often do not focus upon distinguishing characteristics favored by many field ecologists and more user-friendly field guides. This makes learning to identify fish difficult for Ichthyology students. Students usually supplement the use of the key with other methods such as making personal notes, drawings, annotated fish images, and more recently, fish information websites, such as Fishbase. Although these approaches provide useful additional content, it is dispersed across heterogeneous sources and can be tedious to access. Also, most of the existing electronic tools have limited support to manage user created content, especially that related to parts of images such as markings on drawings and images and associated notes. We present SuperIDR, a superimposed image description and retrieval tool, developed to address some of these issues. It allows users to associate parts of images with text annotations. Later, they can retrieve images, parts of images, annotations, and image descriptions through text- and content-based image retrieval. We evaluated SuperIDR in an undergraduate Ichthyology class as an aid to fish species identification and found that the use of SuperIDR yielded a higher likelihood of success in species identification than using traditional methods, including the dichotomous key, fish web sites, notes, etc.
\end{abstract}

Keywords: superimposed information, image annotation, image retrieval, fish, species identification, biodiversity, user study.

\section{Introduction}

Identification of fish species is critical to study of fish ecology and management of fisheries, and follows from precise observation of external morphology, 
coloration, and internal characters. Fish species identification is used in many important tasks such as stream water assessment, where the presence (or absence) of species and their number determine the quality of the stream water. However, learning to correctly identify fishes is difficult for Ichthyology students, who often find traditional dichotomous keys intimidating. Dichotomous keys (Figure 1-a) consist of questions about morphological features of a specimen. Questions are presented in pairs called couplets, and one member of a couplet should describe the specimen in question. Depending on which member of the pair is appropriate, the user will be directed to further questions until, after traversing through a series of couplets, the specimen is identified. For example, if the specimen lacks paired fins and a jaw and has seven gill openings on each side, then it is a lamprey (a family of fishes), and the user is directed to questions to identify the species of that lamprey. If it has paired fins, a jaw, and a single gill opening, then it will belong to any of a number of families, and the user will be asked other questions to identify the family.

However, such keys are incapable of adapting or changing to meet different fish identification approaches, and do not accommodate the range of learning styles actually utilized by students. In particular, keys often do not focus upon distinguishing characteristics favored by many field ecologists and more userfriendly field guides (e.g., Page and Burr 1991 [1]).

\begin{tabular}{|c|c|}
\hline 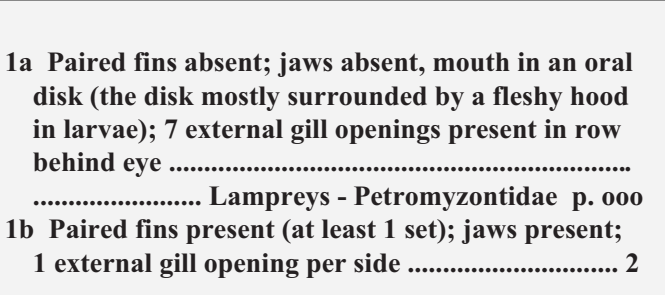 & 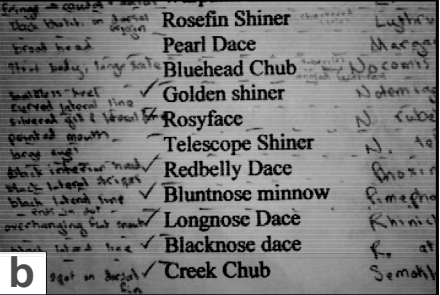 \\
\hline 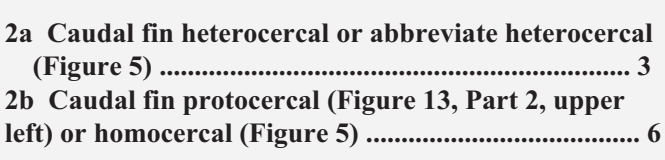 & 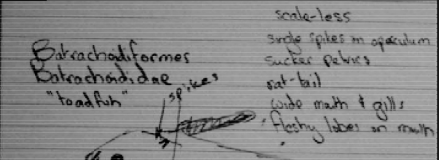 \\
\hline a & \\
\hline
\end{tabular}

Fig. 1. Fish species identification methods. a) Snippet of a dichotomous key; b) Printed list of species superimposed with notes by student on distinguishing characteristics; c) Marked diagram of a fish, indicating distinguishing characteristics, along with other details of the fish, on a notecard.

In addition to the key, students use a variety of other artifacts and tools to study species identification, such as:

- Paper-based artifacts and tools: dichotomous keys, personal notes, notecards, textbooks, field guides, marked drawings, annotated printed pictures of fish, printed lists of families, genera, species, etc. 
- Electronic artifacts and tools: images from Google, Yahoo! search, images on a PowerPoint presentation or Word document, FISHBASE [2], EFISH [3], EKEY [4] ${ }^{1}$, etc.

Almost always a student uses a combination of these artifacts and tools. For example, a student may use digital pictures of fish specimens organized in a PowerPoint presentation. He might then make notes in writing (or typed notes) corresponding to the fish pictures on the slides. Another example is the use of notecards (Figure 1-c). On one side of the notecard, a student might sketch fish diagrams, annotate them with the distinguishing characteristics, and on the other side write a description of the fish. While studying fish species, students typically move back and forth among various artifacts and tools and a preserved specimen, all the while trying to memorize distinguishing characteristics, scientific names, and other information about the fish.

Although the aforementioned methods work for students, they tend to be tedious, time-consuming, and sometimes unsuccessful. Some practical problems are challenging technical terms used in dichotomous keys, insufficient visual or descriptive information for definitively answering questions posed in a dichotomous key, absence or limited variety of reference specimens, inability to share information or identification problems with others not physically present, and the tediousness of accessing information (images, descriptions, markings, notes, etc.).

\subsection{Better Information Management and Access}

The above overview on fish species identification indicates that electronic support for learning to identify fish species is limited and can be improved considerably. There are many issues to consider, including better support for:

- describing and accessing fish images

- managing and accessing user created content, especially that related to parts of images such as markings on drawings and images and associated notes

- sharing this information with others

- providing all of the above listed capabilities in a well-integrated solution

We chose to focus on providing better support to manage and access parts of images and related information. Several domains require scholars to work with images with a significant number of details. In the past, paper-based tools and techniques to work with such information have been used with a fair amount of ease and success. Although the electronic world has provided us with several advantages over paper, such as ease of editing, ease of sharing, and better ability to store, organize, and access information (searching, browsing, etc.), yet the electronic tools used to support working with parts of images are usually not well integrated or do not interoperate well, leading to ineffective and inefficient task execution.

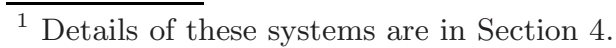


We developed SuperIDR, an image description and retrieval tool, which addresses some of these questions by enabling the user to add content in the form of image markings and annotations, and providing improved image search using text- and content-based image retrieval. Evaluation of SuperIDR in an undergraduate Ichthyology class showed that students identified more unknown fish specimens correctly with SuperIDR than with traditional methods (dichotomous keys, notecards, fish web sites, etc.).

\section{A Superimposed Image Description and Retrieval Tool (SuperIDR)}

We developed SuperIDR, a superimposed image description and retrieval tool, with the aim of helping users to work with parts of images in situ - i.e., being able to select, annotate, retrieve, and share parts of images in the context of the original image. The basis for the functionality was a result of combining Superimposed Information on images along with Content-Based Image Retrieval.

Superimposed information (SI) refers to new information laid over existing information (such as bookmarks, annotations, etc.) [5]. Superimposed applications (SAs) allow users to lay new interpretations over existing or base information. SAs employ "marks", which are references to selected regions within base information. SAs enable users to (a) deal with information of varying granularity, and (b) select or work with information elements at sub-document level while retaining the original context. In SuperIDR, we worked with image marks, or references to parts of fish images such as fin, mouth, body, tail, etc., in the context of the entire fish image.

Content-Based Image Retrieval (CBIR) systems aim to retrieve images similar to a user-defined specification or pattern based on content properties (e.g., shape, color or texture), usually encoded into feature vectors [6]. We use the Content-Based Image Search Component (CBISC), an Open Archives Initiative (OAI)-compliant component that provides an easy-to-install search engine to query images by content [7]. It can be readily tailored for a particular collection by a domain expert, who carries out a clearly defined set of pilot experiments. It supports the use of different types of vector-based image descriptors, and then easily combines them to yield improved effectiveness. CBISC encapsulates a metric index structure to speed up the search process, which can be easily configured for different image collections. For SuperIDR, we used the .NET version of CBISC [8] to index and retrieve complete images as well as parts of images (defined by image marks).

SuperIDR is an extension of a PC-only version that we developed earlier [9]. It has been developed in C\# and uses MySQL as the database. We developed SuperIDR to work with tablet PCs, taking advantage of pen-based input. We felt that this would emulate, as close as possible, the use of a pen on paper, which many biologists are used to since they make markings often. Also, we felt that when used in the field, it would be more convenient to work with pen input versus using a touchpad or keypad. For example, a fisheries scholar could use the pen-input to mark a feature on a fish image and then write notes describing that mark. 
SuperIDR is seeded with details of 207 species of freshwater fishes of Virginia, taken from [10]. Each species has a representative image as shown in Figure 2-b. In addition to making annotations, SuperIDR allows searching and browsing of species descriptions, images, image marks, and annotations. A user can search in one of two ways: 1) perform text-based search (full-text and field-wise search, powered by Lucene.NET ${ }^{2}$ ) on species descriptions and annotations, where the query may include terms, phrases, or their boolean combination; 2) perform content-based image search on images and annotated-image-marks, where the query could be a complete image or part of an image. Finally, in SuperIDR, a user can browse through species information either through a taxonomic organization of species based on family and genera or through an electronic version of the dichotomous key. The scenario described below illustrates how an Ichthyology student would use SuperIDR.

Scenario: Matt is a junior, majoring in Fisheries, enrolled in the Ichthyology class. He has some experience with species identification but still is quite intimidated by dichotomous keys. In the past, he has supplemented the use of the keys with personal notes, pictures from the web, textbooks, etc. The Ichthyology teacher has decided to use a new software tool in class, SuperIDR. Matt is moderately familiar with computers and is enthusiastic about using SuperIDR, as it will help him with species identification.

Matt walks through the various features of SuperIDR, beginning with a taxonomy browser, where all species have been organized according to families and then genera. He browses to one of this week's species, the redear sunfish (Figure 2-a). The species description screen provides him details about the fish, including physical description, habitat, food habits, etc. (Figure 2-b). However, it does not have distinguishing characteristics explained in detail, which are an important part in identifying the fish species. Matt adds a new annotation using a pen input. He marks the fish picture and associates the marks with text explanations, notes in Matt's own words, making it easier for Matt to remember and learn about this species. He makes several such annotations on the different fish images about which he is learning (Figure 2-c).

In two weeks, Matt's teacher holds a practice specimen identification test. Some of the unknown specimens are in jars and some are in the form of images posted on the course web site. Matt examines the fish specimens for specific characteristics and uses text search on annotations and species descriptions (Figure 2-d, e). Browsing through the result list and occasionally clicking on a result for details, he is able to narrow down the options to the species of the unknown specimen. Sometimes he uses the electronic key available in SuperIDR. For some images of unknown fishes, Matt uses the content-based image search feature. He vaguely has some idea about the fish, but does not remember the exact keywords. He can search on all or part of the fish image content and browse through the result list as he would do with the text search results (Figure 2-f, g). Using SuperIDR not only makes it faster for Matt to identify the species of the unknown specimens but also reinforces descriptions of those species through distinguishing characteristics.

\footnotetext{
$\overline{2}$ http://incubator.apache.org/lucene.net/
} 


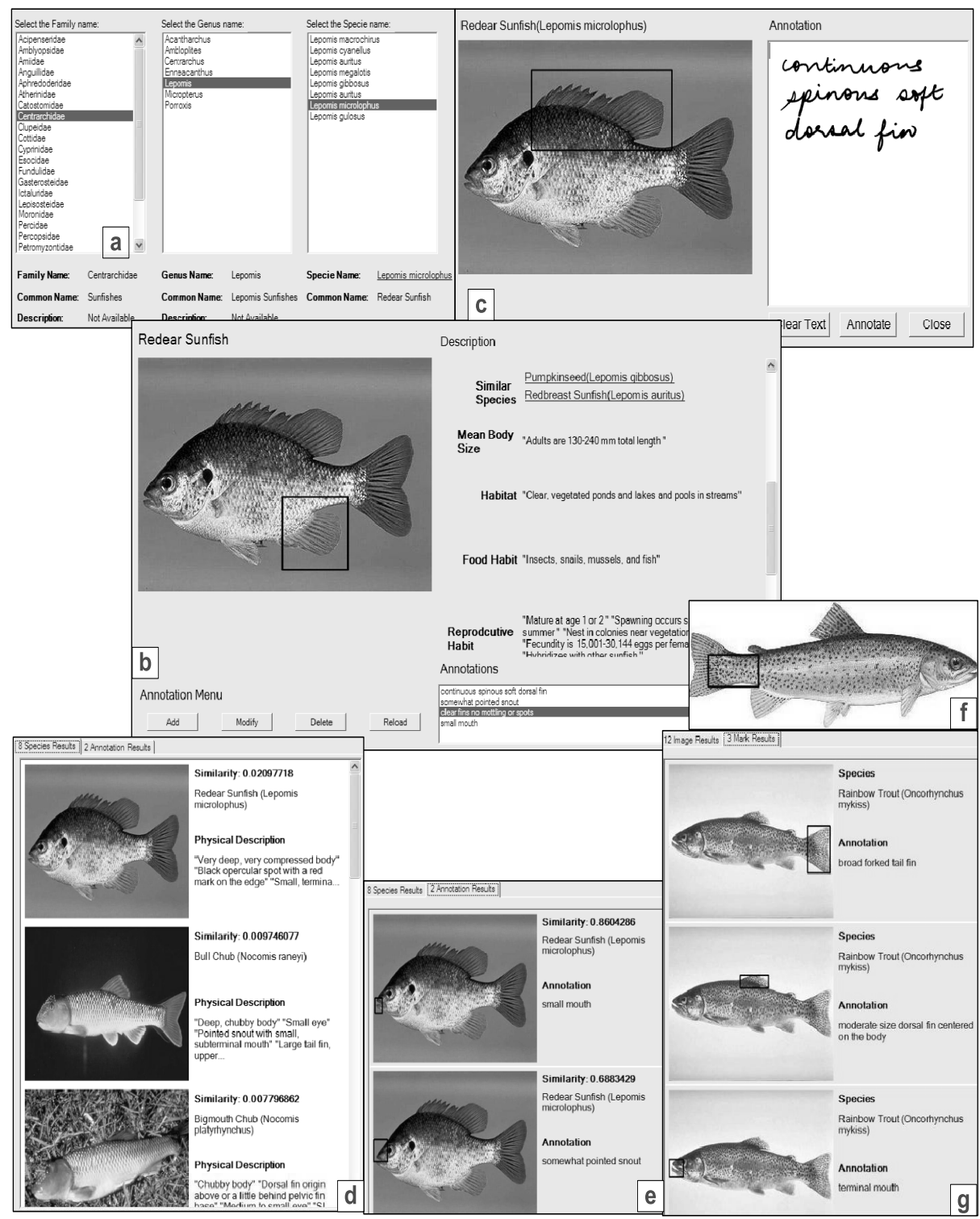

Fig. 2. Screenshots of SuperIDR features: a) Taxonomy browser; b) Species description screen shows details of species and annotations - the highlighted annotation (bottom right) is associated with a marked region in the image; c) Annotation screen - pen input is used to mark the fish image and "write" the annotation, which gets automatically recognized; d) Eight species description results for the text query " "red mark" "small mouth" "pointed snout" "no spots"'; e) Two annotation results for the same text query; f) Content-based image search, where the query is the marked region that covers black dots on the body of a rainbow trout; g) Image search results, which can be annotated image marks (shown in the figure) and/or complete images. 


\section{Classroom-Based Evaluation}

We had several interactions with fisheries students, faculty, and researchers to help with development and improvement of SuperIDR. In a two-month-long longitudinal formative evaluation with five fisheries scholars (three students, one faculty member, and one researcher), we made improvements and added new features to SuperIDR, including replacing database indexing with full text indexing of species descriptions and annotations, adding user controls for better management of annotations, and fixing bugs.

In order to assess the effectiveness of SuperIDR as an aid to species identification, we conducted an experiment in an undergraduate Ichthyology class. In the experiment, we compared the use of SuperIDR with traditional methods of species identification (either by using the key, personal notes, markings on images, use of websites, notecards, etc., or a combination of the aforementioned methods). Most students in that class were juniors in Fisheries Science. Participation in the evaluation study was voluntary and students' performance on the quiz (which was part of the experiment) was not included in their final grades.

There were 28 students in the class and all were present in the first meeting of the experiment. However, we considered data from only 18 of them since the remaining either did not show up for the remaining meetings or we had incomplete data from them. Seventeen of the 18 students rated themselves as moderately or very familiar with computers although 13 of them indicated that they had very low expertise with tablet $\mathrm{PCs}^{3}$. Sixteen students had previously taken a course on species identification (not necessarily fish species), so most of them were familiar with the task. Most students used annotations of some form on paper for learning, but most of them did not use digital annotation systems.

We conducted the experiment in April 2008, in the 12th week of a 15-weeklong class. SuperIDR was set up on tablet PCs. Due to limited availability of tablet PCs, we asked the students to form teams of two (based on their seating in class that day) and share a tablet PC. Students were given a tutorial on the tool as well as a user manual for later reference. We asked the students to use the tool for the week, explore its features and make several annotations on images of the species they had studied, in order to prepare for a test the following week.

In a lab period in the following week, we had two sessions - session 1 and session 2. We divided the class (consisting of teams) into two parts - A and B. In each session, students working in teams had to identify 20 unknown specimens. In session 1, teams in part A used only SuperIDR as an aid to species identification, and teams in part B used traditional methods. In session 2, teams in part A used traditional methods and teams in part B used only SuperIDR.

In the experiment, we collected the following data:

- Species identification responses for the 40 unknown specimens: For a specimen to be correctly identified, its family, genus, and scientific name had to be correctly identified. We computed a team's score, i.e., the number of correctly identified specimens, for each session.

\footnotetext{
${ }^{3}$ For these questions, students rated themselves on a scale of 1 to 5 with 1 being least familiar and 5 being very familiar.
} 
- Entry (demographic data and data about prior experience with species identification and software tools) and exit questionnaire responses (qualitative feedback on the use of SuperIDR)

- Log data of user interaction with SuperIDR

\subsection{Experiment Data Analysis}

Species Identification Responses: Table 1 provides a summary of the scores for teams using different methods across the two sessions. In the experiment, each team worked with one specimen at a time, and three main factors impacted the outcome - correct or incorrect - of a species identification task: 1) the nature of the specimen; 2) the team (of two students) working on it; and 3) the method used to identify it - the tablet PC tool method or traditional method. Keeping this in mind, we used the generalized linear model with a binomial (logit) link function to analyze the species identification responses. Generalized linear models, an extension of the linear modeling process, allow models to be fit to data that follow probability distributions other than the Normal distribution (such as the Poisson or the Binomial distribution) [11].

Statistical analysis using $\mathrm{R}^{4}$ showed that the the team (p-value=0.015) and the method ( $\mathrm{p}$-value $=0.011$ ) had a significant impact on the outcome of the species identification task, while the session (hence, the nature of specimen) did not impact the outcome significantly. The mean values in Table 1 show that using SuperIDR yielded a higher likelihood of identifying a specimen correctly than using traditional methods.

Exit Questionnaire Responses ${ }^{5}$ : Students gave their feedback on the tool through an exit questionnaire. In general, students' knowledge of computerassisted fish identification improved during the course of the study. Students still preferred the use of the key (10) versus SuperIDR (6) (two students didn't respond to this question). One reason for this could be the timing of the study, which was towards the end of the semester. At that time, students already had established practices for species identification. Most were busy practicing their known skills for upcoming exams, and did not spend too much time on the tool (also indicated in the log data). Another reason for the students' preference was that many were frustrated with the pen input. Although we felt that it might have been useful, students were frustrated because of the poor ink response (slow and distorted sometimes) and the recognition of handwriting. Students also felt that the number and quality of images was low. This was a serious drawback since there was no support for students to add their fish pictures or diagrams to the existing image collection. Some of these reactions are reflected in the students' comments:

- "Very neat but may take a while to master all the key concepts."

- "It was very helpful."

\footnotetext{
${ }^{4}$ http://www.r-project.org/

5 The entry questionnaire responses have been summarized above, where information about the participants is given.
} 
Table 1. Number of correct responses, from 20 specimens, of different teams using traditional methods and using SuperIDR

\begin{tabular}{|c|c|c|c|c|c|}
\hline \multicolumn{3}{|c|}{ Traditional Methods } & \multicolumn{3}{|c|}{ SuperIDR } \\
\hline Team ID & Session & Correct & Team ID & Session & Correct \\
\hline 2 & 1 & 15 & 2 & 2 & 18 \\
\hline 4 & 1 & 16 & 4 & 2 & 17 \\
\hline 6 & 1 & 13 & 6 & 2 & 17 \\
\hline 11 & 1 & 12 & 11 & 2 & 16 \\
\hline 3 & 2 & 8 & 3 & 1 & 14 \\
\hline 5 & 2 & 13 & 5 & 1 & 15 \\
\hline 9 & 2 & 12 & 9 & 1 & 10 \\
\hline 10 & 2 & 10 & 10 & 1 & 14 \\
\hline 13 & 2 & 11 & 13 & 1 & 11 \\
\hline \multicolumn{2}{|c|}{ Mean } & 12.2 & \multicolumn{2}{|c|}{ Mean } & 14.67 \\
\hline
\end{tabular}

- "Very helpful for taxonomy, still needs better photos."

- "If you had started the program at the beginning of the semester, there would be higher success and better likelihood that we may use it."

Log Data: Log data showed that students used the Browse feature the most (logged 2450 times) and made 500 search requests with 99 being image searches and 451 being text searches. We were very interested in knowing how students make use of image marks and of superimposed information in species identification, through both annotation and searching. However, there was no record of use of the Annotation feature in the log data. The reason for this (as also mentioned above) is the timing of the experiment. Most students used it only on the two days they came to class for the experiment.

\subsection{Evaluation Summary}

Overall, we believe that SuperIDR was well received by Fisheries scholars as an aid to species identification. Students identified more specimens correctly with SuperIDR than with traditional methods, with just a week of use. The questionnaire responses indicated a preference for traditional methods versus SuperIDR and the log data did not record activity on the annotation front. As mentioned earlier, we think this was because of the timing and duration of the experiment. One more indication of student interest in the tool is that six out of the fourteen teams chose to keep SuperIDR for three more weeks till the end of the semester. Students said that they wanted to explore the tool further. In addition to the results mentioned, we received several suggestions for extensions and improvements to the tool.

\section{Related Work}

Recently, there have been systems and tools developed to support biodiversity researchers and scholars. For example, Lyons et al. [12] described a photo-based 
computer system for identifying Wisconsin fishes that features multiple images of each species which can be accessed by dichotomous key, a query tool, and a slide show. EcoPod is a PDA-based application, which replaces traditional paper field guides with a mobile computing platform [13]. It focuses on enabling users to work with keys to identify species in the field. It has limited support for adding user content and also does not have any CBIR capabilities. In [14], the authors achieve a high level of accuracy in automatically identifying moth species using data mining techniques. However, their system does not provide any text annotation or retrieval capabilities.

Other systems include the popular online FishBase [2], an information system providing information to fisheries professionals on the 31,000 known species of fishes. Information access in FishBase is limited to browsing and field-wise searching. FishBase also provides forums to discuss problems with other scholars. EFish [3] is another system providing species identification and life history information for 200 species of freshwater fishes of Virginia. Information access in EFish is limited to browsing and there is no facility to add user content. EKEY [4], a predecessor to SuperIDR, is a web-based system with an electronic dichotomous key, a taxonomy browser and provides text- and shape-based search. SuperIDR builds upon the features of these systems, while enabling the user to add content to the existing information base. Also, it provides support for working with specific parts of images and performing content-based image description and retrieval. In addition, it has pen-input capabilities, mimicking free-hand drawing and writing on paper.

Many digital libraries have annotation capabilities, usually focusing on annotations of complete documents. There has been work done to provide annotation support for image digital libraries such as [15], [16]. Also, there are several photo annotation systems, including online tools such as Flickr ${ }^{6}$ and Fototagger ${ }^{7}$. However, most of these systems do not combine the capabilities to work with textand content-based description and retrieval of parts of images. With regard to integrating subdocuments with digital libraries, we developed an architecture for representing SI in DSpace ${ }^{8}[17]$. The focus of that work was mainly on text documents, with limited support for images.

\section{Discussion and Future Work}

We presented SuperIDR, a tool that combines text- and content-based image description and retrieval, as an aid to fish species identification. Students performed well with SuperIDR and it was generally well received by fisheries students, faculty, and researchers.

Our initial goal was to provide support for scholars to work with images with significant numbers of details such as those in Fisheries Sciences. Through field trips, in-class observations, and interactions with fisheries scholars, we found

\footnotetext{
${ }^{6}$ http://flickr.com

7 http://www.fototagger.com/

${ }^{8}$ htp://dspace.org
} 
that working with parts of images is important for fish species identification. Some reasons for this are: 1) distinguishing characteristics usually focus on part of the fish (and fish image); 2) students work with (annotate, browse, and search) parts of images in their notes, online information, and keys, while studying and while identifying fishes; 3) they frequently go back and forth between notes and marked fish images; and 4) they compare parts of different fish images to study differences between species, genera, and families. However, we did not find any significant evidence of such use from our experiments (log data). As mentioned earlier, we believe this was due to the timing and duration of our experiment.

We feel that we need to understand and provide better digital support for scholars to work with parts of images in the digital world. Towards this, we are conducting in-depth interviews with the students who participated in the classroom experiment. We have conducted four such interviews and have received more details on use of SuperIDR and on working with parts of images. For example, one student said that SuperIDR would be useful in any field with an "-ology" suffix. Another student said that the most useful features in SuperIDR with regard to species identification are working with images and adding personal notes to images.

We have made further improvements to SuperIDR based on feedback received from the user studies, and will be making it available to download to fisheries scholars. A natural extension of this system is to make it web-enabled, thus supporting content-sharing across image management systems and social networks.

We used fish species identification as the specific scholarly task to situate our research ideas. However, we believe that our work is applicable to any scholarly task/domain involving images with a significant number of details, such as analyzing paintings in art history, examining a building style in architecture, understanding trees in dendrology, etc.

\section{Acknowledgments}

Thanks go to Microsoft (under a tablet PC grant) for funding this project. We also thank our previous sponsors who helped initiate this work including NSF (DUE-0435059), CAPES, FAPESP, FAEPEX, and CNPq. We especially thank Dr. Donald Orth, Ryan McManamay, Lindsey Pierce, and all the participants in the evaluations. Many thanks go to Jason Lockhart and the College of Engineering at Virginia Tech for loaning us tablet PCs to conduct experiments. We are grateful to the American Fisheries Society for use of copyrighted material from Jenkins and Burkhead's Freshwater Fishes of Virginia.

\section{References}

1. Page, L., Burr, B.: A Field Guide to Freshwater Fishes: North America North of Mexico (Peterson Field Guides). Houghton Mifflin Co., New York (1991)

2. WorldFishCenter: Fishbase: A global information system on fishes (1997), http://www.fishbase.org/home.htm 
3. Helfrich, L., Newcomb, T., Hallerman, E., Stein, K.: Efish: The virtual aquarium (2001), http://www.cnr.vt.edu/efish/

4. da, S., Torres, R., Hallerman, E., Jenkins, R.E., Burkhead, N.M., Herrington, B.: Ekey - the electronic key for identifying freshwater fishes (2004), http://fwie.fw.vt.edu:8080/ekey/

5. Maier, D., Delcambre, L.M.L.: Superimposed information for the internet. In: WebDB (Informal Proceedings), pp. 1-9 (1999)

6. da, S., Torres, R., Falcão, A.X.: Content-based image retrieval: Theory and applications. Revista de Informática Teórica e Aplicada 13(2), 161-185 (2006)

7. da, S., Torres, R., Medeiros, C.B., Goncalves, M.A., Fox, E.A.: A digital library framework for biodiversity information systems. International Journal on Digital Libraries 6(1), 3-17 (2006)

8. Kozievitch, N.P., Falcão, T.R.C., da, S., Torres, R.: A .Net implementation of a content-based image search component. In: Demo Session, 23th Brazilian Symposium on Databases, Campinas, Brazil (2008)

9. Murthy, U., da Silva Torres, R., Fox, E.A.: Sierra - a superimposed application for enhanced image description and retrieval. In: Gonzalo, J., Thanos, C., Verdejo, M.F., Carrasco, R.C. (eds.) ECDL 2006. LNCS, vol. 4172, pp. 540-543. Springer, Heidelberg (2006)

10. Jenkins, R.E., Burkhead, N.M.: Freshwater Fishes of Virginia. American Fisheries Society, Bethesda (1994)

11. McCullagh, P., Nelder, J.A.: Generalized linear models. CRC Press, Boca Raton (1989)

12. Lyons, J., Hanson, P., White, E.: A photo-based computer system for identifying wisconsin fishes. Fisheries 31(6), 269-275 (2006)

13. Yu, Y., Stamberger, J.A., Manoharan, A., Paepcke, A.: EcoPod: a mobile tool for community based biodiversity collection building. In: JCDL 2006: Proceedings of the 6th ACM/IEEE-CS Joint Conference on Digital Libraries, pp. 244-253. ACM Press, New York (2006)

14. Mayo, M., Watson, A.T.: Automatic species identification of live moths. Knowledge-Based Systems 20(2), 195-202 (2007)

15. Jochum, W., Kaiser, M., Schellner, K., Wirl, F.: Living memory annotation tool image annotations for digital libraries. In: Kovács, L., Fuhr, N., Meghini, C. (eds.) ECDL 2007. LNCS, vol. 4675, pp. 549-550. Springer, Heidelberg (2007)

16. Stein, A., Thiel, U., Brocks, H.: COLLATE - collaboratory for annotation, indexing and retrieval of digitized historical archive material (2004),

http://www. collate.de/

17. Archer, D.W., Delcambre, L.M., Corubolo, F., Cassel, L., Price, S., Murthy, U., Maier, D., Fox, E.A., Murthy, S., Mccall, J., Kuchibhotla, K., Suryavanshi, R.: Superimposed information architecture for digital libraries. In: ChristensenDalsgaard, B., Castelli, D., Ammitzbøll Jurik, B., Lippincott, J. (eds.) ECDL 2008. LNCS, vol. 5173, pp. 88-99. Springer, Heidelberg (2008) 